\title{
Prevalence of allergen sensitization among 15,534 patients with suspected allergic diseases in Henan Province, China
}

\author{
Xiaoxu Sun, ${ }^{16}$ Junwei Zhao, ${ }^{16}$ Qiuya Wang, ${ }^{2}$ Guang Shi, ${ }^{1}$ Jingjing Yang, ${ }^{1}$ Liang Ming ${ }^{1 *}$
}

\begin{abstract}
Background: The prevalence of allergen sensitization varies in different geographic areas and population subsets. This study investigated the prevalence and distribution of inhaled and food allergens among allergic patients in Henan Province, China.

Method: The prevalence and distribution of 19 inhaled and food allergens among 15,534 patients with suspected allergic diseases were retrospectively analyzed in Henan Province, China between December 2012 and August 2016. Reactivity of their serum-specific immunoglobulin E (sIgE) to allergens was tested using the AllergyScreen test.

Results: Of the 15,534 patients tested, the sIgE of 6520 (41.97\%) was tested positive for at least one of the 19 tested allergens. Dermatophagoides pteronyssinus (3540, 22.79\%), cockroach (1398, 9.00\%), and mold mix (1301, 8.38\%) were the most frequently tested inhaled allergens, while cashew nut $(1320,8.50 \%)$, mango $(768,4.94 \%)$ and cow's milk $(748,4.82 \%)$ were the most frequently tested food allergens. The overall sIgE positive rate was higher in males than that in females $(p<$ 0.05). Moreover, the sIgE positive rates to the most of the inhaled and food allergens were statistically different between age groups $(p<0.05)$. The sIgE positive rates of the main inhaled allergens increased with age. However, the sIgE positive rates for the primary food allergens were higher in the younger groups and lower in the older groups.

Conclusions: The characteristics of allergen sensitization revealed in this study in Henan Province would facilitate the prevention, diagnosis, and management of allergic diseases in this region.
\end{abstract}

Keywords: Allergen; Prevalence; specific IgE; Henan, China

From:

${ }^{1}$ Department of Clinical Laboratory, The First Affiliated Hospital of Zhengzhou University, Key Clinical Laboratory of Henan Province, Zhengzhou, 450052, Henan, China

2 Department of Hospital Infection Management, The First Affiliated Hospital of Zhengzhou University, Zhengzhou, 450052, Henan, China

¿ These authors contributed equally to this work.

\section{Background}

Allergic diseases are chronic, immune-mediated diseases including allergic rhinitis, allergic asthma, atopic dermatitis, and food allergy. The clinical manifestations of allergic diseases include runny nose, red or itchy eyes, skin rash, hives, eczema, sneezing, shortness of breath which can seriously affect quality of life. Over the past few decades, the prevalence and incidence of allergic diseases have increased at different rates, ${ }^{1-3}$ and they are associated with substantial medical and economic burdens to these patients. ${ }^{4,5}$

Previous studies have demonstrated that both genetic and environmental factors play critical roles in the genesis and development of allergic diseases ${ }^{6,7}$ Therefore, the prevalence of

\author{
* Corresponding author: \\ Liang Ming \\ Department of Clinical Laboratory, The First Affiliated Hospital of \\ Zhengzhou University, Key Clinical Laboratory of Henan Province, 1 \\ Jianshe East Road, Zhengzhou, 450052, Henan, China \\ E-mail: mingliang3072@163.com
}

allergen sensitization may vary between different geographic areas, local climates, lifestyles, and population subsets. Data regarding local epidemiology of allergens are vital to support evidence-based prevention and management strategies. However, there was no local epidemiological data on the prevalence of sensitization to allergens among patients with allergic diseases in Henan Province, the central region of China over the past ten years.

Several techniques have been investigated in recent years to identify specific allergens for allergic diseases. Apart from skin prick test, the serum-specific IgE test would be an attractive alternative method to identify specific allergens test, ${ }^{8,9}$ which 
is rapid, easy to perform and user friendly. In this study, we retrospectively analyzed the allergen sensitivity status of 15,534 patients with suspected allergies who underwent the Allergy Screen test for SIgE between December 2012 and August 2016 in Henan Province, China, to facilitate the prevention, diagnosis, and management of allergic diseases in this region.

\section{Methods \\ Patients}

In this retrospective study, the medical records of 15,534 consecutive patients with suspected allergy who sought medical care at the First Affiliated Hospital of Zhengzhou University, Henan Province, China, between December 2012 and August 2016 were analyzed. These patients were clinically evaluated by their attending physicians. All patients had suspected symptoms of allergies including runny nose, red or itchy eyes, skin rash, hives, eczema, sneezing, shortness of breath. Patients with other diseases had been excluded in this study.

\section{The AllergyScreen test}

The AllergyScreen test (Mediwiss Analytic GmbH, Moers, Germany), an immunoblot assay, was used to semi-quantitatively detect circulating allergen-specific immunoglobulin $\mathrm{E}$ (IgE) in human serum.

Allergens tested using this system include 10 inhaled allergens (Dermatophagoides pteronyssinus, house dust, mulberry, dog hair, cat dander, cockroach, amaranth, mold mix, grass mix, and tree pollen mix) and 9 food allergens (egg, cow's milk, shrimp, beef, cowry, crab, mango, cashew nut, and pineapple).

AllergyScreen tests were performed as below. Special allergens are bound to the surface of nitrocellulose membranes lying in a reaction trough. when the patient's serum is pipetted into the reaction trough and incubated, the allergen -specific IgE-antibody react with the allergens, after washing an anti-human IgE antibody coupled with biotin is added and incubated. Non-bound detector antibodies are removed by washing. Next a streptavidin is added which is conjugated with alkaline phosphatase to bind the biotin from the second incubation. Non-bound streptavidin conjugate is removed by washing. After adding the substrate and incubated, a specific enzymatic color reaction of the alkaline phosphatase takes place which results in the formation of precipitates on the strips. The coloration is directly proportional to the specific antibody content of the serum sample. Evaluation is carried out after complete drying of the test strip with the Rapidreader, which take a photo of the membrane and a software programme evaluates the colouration of the allergen lines.

The results of the AllergyScreen test were semi-quantified and categorized into the following groups: class $0(<0.35 \mathrm{IU} /$ $\mathrm{mL})$, class $1(0.35-0.70 \mathrm{IU} / \mathrm{mL})$, class $2(0.70-3.50 \mathrm{IU} / \mathrm{mL})$, class $3(3.5-17.5 \mathrm{IU} / \mathrm{mL})$, class $4(17.5-50 \mathrm{IU} / \mathrm{mL})$, class $5(50-100$ $\mathrm{IU} / \mathrm{mL})$, class $6(>100 \mathrm{IU} / \mathrm{mL})$. The cut-off value was set as $0.35 \mathrm{IU} / \mathrm{mL}$, sIgE $\geq 0.35 \mathrm{IU} / \mathrm{mL}$ was considered positive.

\section{Statistical Analysis}

All data were analyzed using the Statistical Package for Social Sciences version 13.0 (SPSS Inc., Chicago, IL, USA). The chi-squared test or Fisher's exact probability method was used to evaluate differences between groups of categorical variables. $P<0.05$ was considered statistically significant.

\section{Results \\ Characteristics of the study population}

The study population consisted of 7388 males (47.56\%) and 8146 females (52.44\%), including 5257 children (33.84\%) and 10277 adults (66.16\%). The patient age ranged from $1-95$ years, the average age was $30.56 \pm 20.98$ (mean \pm SD) years. $44.6 \%$ of the patients come from towns and $55.4 \%$ of the patients come from rural areas.

\section{Overall allergen sensitization to inhaled and food allergens}

Of the 15,534 patients, 6520 (41.97\%) tested positive for sIgE to at least one of the 19 allergens. D. pteronyssinus (3540, $22.79 \%)$, cockroach $(1398,9.00 \%)$ and mold mix (1301, 8.38\%) were the top three inhaled allergens, while cashew nuts (1320, $8.50 \%)$, mango $(768,4.94 \%)$ and cow's milk $(748,4.82 \%)$ were the most frequently tested food allergens (Table 1).

Table 1. Overall allergen sensitization to inhaled and food allergens.

\begin{tabular}{|c|c|c|c|}
\hline Allergens & & Patients(N) & $\%$ \\
\hline \multirow[t]{10}{*}{ Inhaled } & D. pteronyssinus & 3540 & 22.79 \\
\hline & House dust & 560 & 3.60 \\
\hline & Mulberry & 806 & 5.19 \\
\hline & Cat dander & 275 & 1.77 \\
\hline & Dog hair & 902 & 5.81 \\
\hline & Cockroach & 1398 & 9.00 \\
\hline & Amaranth & 1170 & 7.53 \\
\hline & Mold mix & 1301 & 8.38 \\
\hline & Grass mix & 1029 & 6.62 \\
\hline & Tree pollen mix & 1273 & 8.19 \\
\hline \multirow[t]{9}{*}{ Food } & Egg & 214 & 1.38 \\
\hline & Cow's milk & 748 & 4.82 \\
\hline & Shrimp & 618 & 3.98 \\
\hline & Beef & 114 & 0.73 \\
\hline & Cowry & 52 & 0.33 \\
\hline & Crab & 507 & 3.26 \\
\hline & Mango & 768 & 4.94 \\
\hline & Cashew nut & 1320 & 8.50 \\
\hline & Pineapple & 255 & 1.64 \\
\hline
\end{tabular}

\section{Allergen sensitization in different gender groups}

The allergen sensitization in this study were significantly different between male and female groups $\left(\chi^{2}=48.57, P<0.01\right)$, As shown in Table 2, the overall sIgE positive rate was higher among males than females ( $44.87 \%$ vs. $39.34 \%$,). All allergens, 
Table 2. Allergen sensitization in male and female participants

\begin{tabular}{|c|c|c|c|c|c|c|}
\hline Allergens & Male (N) & $\%$ & Female (N) & $\%$ & Chi square test $\left(\chi^{2}\right)$ & $P$ value \\
\hline D. pteronyssinus & 1788 & 24.20 & 1752 & 21.51 & 15.979 & $<0.01$ \\
\hline House dust & 290 & 3.93 & 270 & 3.31 & 4.159 & 0.041 \\
\hline Mulberry & 463 & 6.27 & 343 & 4.21 & 33.299 & $<0.01$ \\
\hline Cat dander & 173 & 2.34 & 102 & 1.25 & 26.445 & $<0.01$ \\
\hline Dog hair & 509 & 6.89 & 393 & 4.82 & 30.208 & $<0.01$ \\
\hline Cockroach & 792 & 10.72 & 606 & 7.44 & 50.921 & $<0.01$ \\
\hline Amaranth & 658 & 8.91 & 512 & 6.29 & 38.216 & $<0.01$ \\
\hline Mold mix & 575 & 7.78 & 726 & 8.91 & 6.441 & 0.011 \\
\hline Grass mix & 592 & 8.01 & 437 & 5.36 & 43.933 & $<0.01$ \\
\hline Tree pollen mix & 702 & 9.50 & 571 & 7.01 & 31.988 & $<0.01$ \\
\hline Egg & 122 & 1.65 & 92 & 1.13 & 7.768 & $<0.01$ \\
\hline Cow's milk & 463 & 6.27 & 285 & 3.50 & 64.777 & $<0.01$ \\
\hline Shrimp & 322 & 4.36 & 296 & 3.63 & 5.327 & 0.021 \\
\hline Beef & 67 & 0.91 & 47 & 0.58 & 5.788 & 0.016 \\
\hline Cowry & 25 & 0.34 & 27 & 0.33 & 0.006 & 0.940 \\
\hline Crab & 266 & 3.60 & 241 & 2.96 & 5.056 & 0.025 \\
\hline Mango & 431 & 5.83 & 337 & 4.14 & 23.735 & $<0.01$ \\
\hline Cashew nut & 759 & 10.27 & 561 & 6.89 & 57.147 & $<0.01$ \\
\hline Pineapple & 150 & 2.03 & 105 & 1.29 & 13.187 & $<0.01$ \\
\hline
\end{tabular}

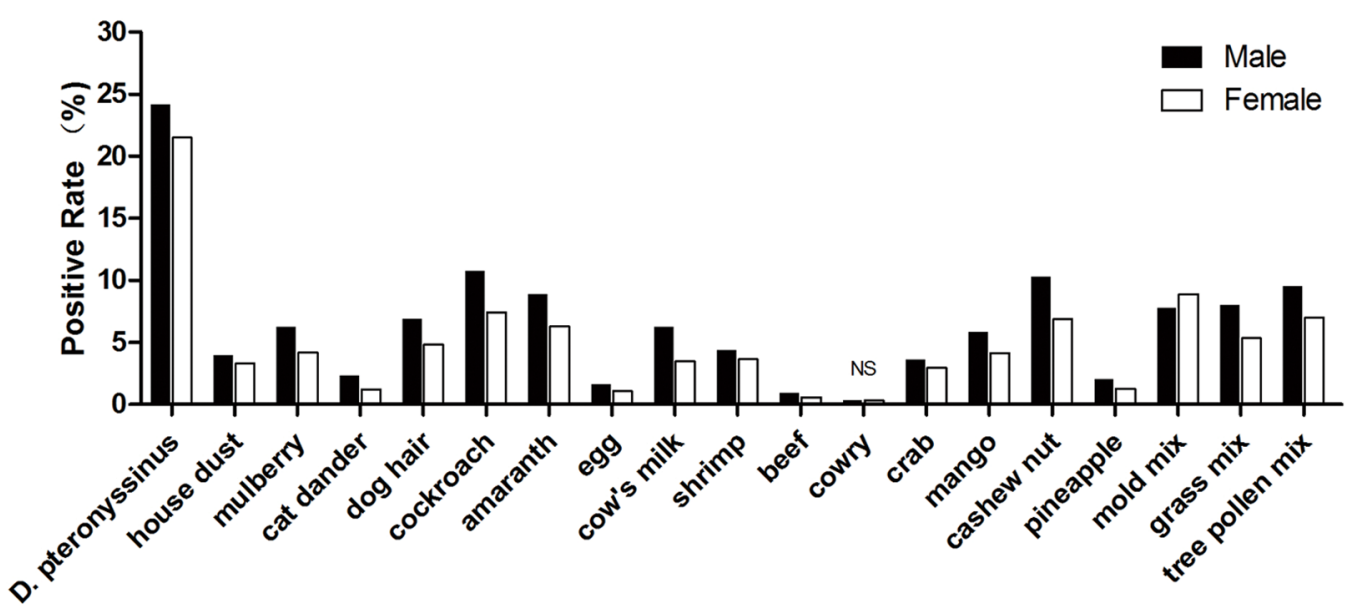

Allergens

Figure 1. Allergen sensitization in different gender groups. NS: no statistically significant difference.

except cowry and mold mix, elicited a higher sIgE positive rate among males than that among females (Figure 1).

\section{Allergen sensitization in different age groups}

Individuals were divided into 10 groups based on age (0-3, $4-6,7-9,10-12,13-15,16-18,19-24,25-40,41-59$, and 60 years and above). As shown in Table 3, The sensitization to any allergen were significantly different among groups $(p<$ 0.01 ). The sIgE positive rates to several inhaled allergens were low in the younger groups, increased with age, peaked, then declined. The rates of positive sIgE response to D. pteronyssinus, house dust, and cockroach peaked in the 19-24 age group (positive rates $34.91 \%, 5.93 \%$, and $14.40 \%$, respectively). But the sIgE positive rates for mulberry, amaranth, grass mix, and tree mix peaked in the $25-40$ age group $(6.88 \%, 9.80 \%, 9.15 \%$, and $10.54 \%$, respectively). However, the sIgE positive rates declined with age thereafter, but remained at a relatively high level. Unlike these inhaled allergens, the sIgE positive rates for 


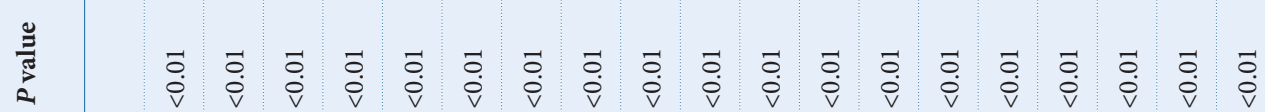
苞華 色

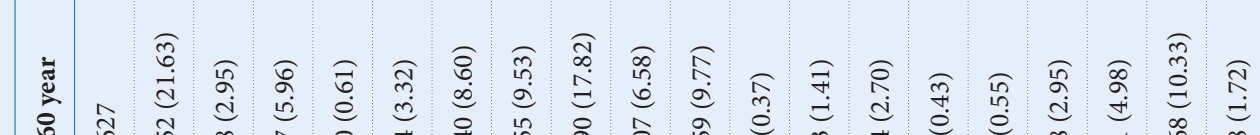

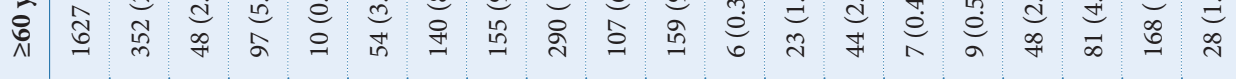

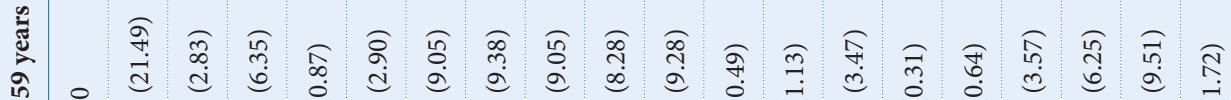

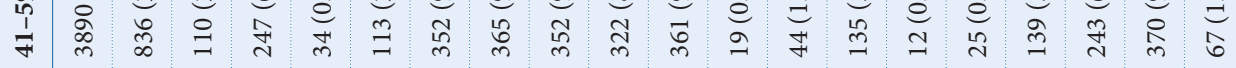

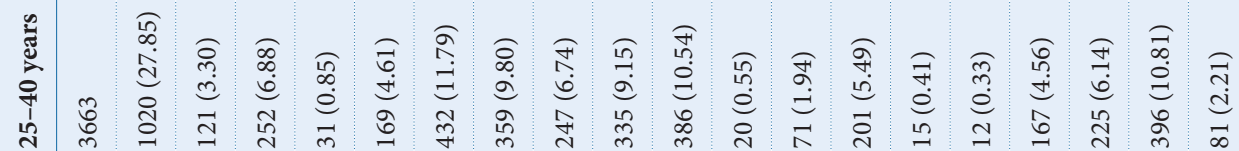

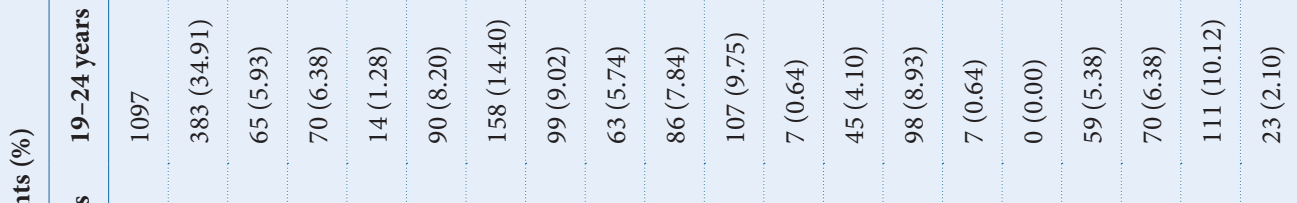
:

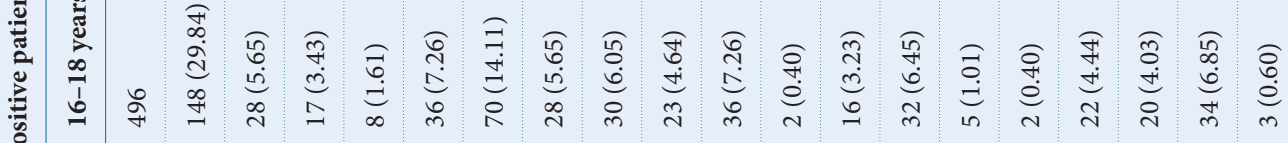
ฉ.

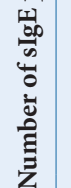

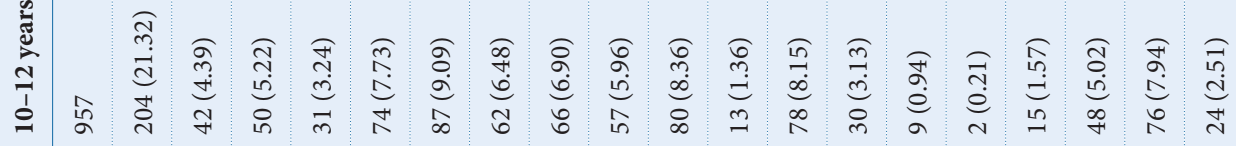

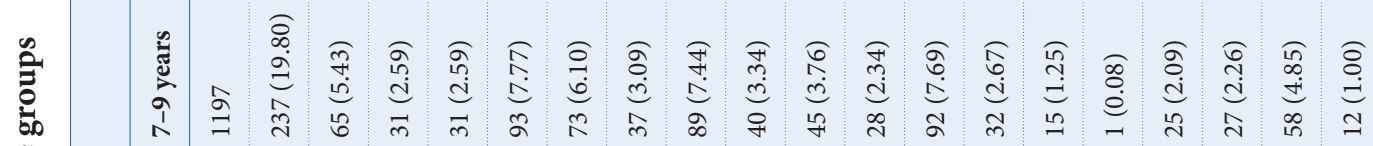

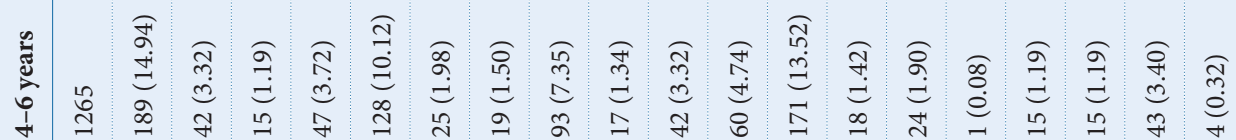

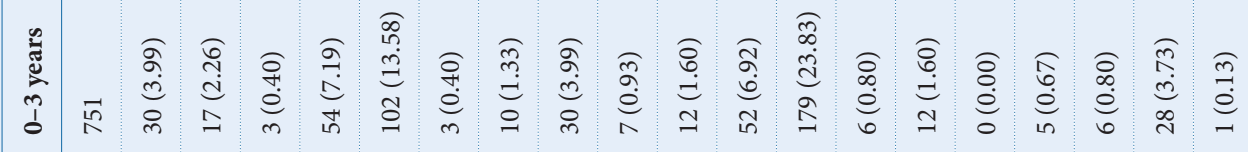



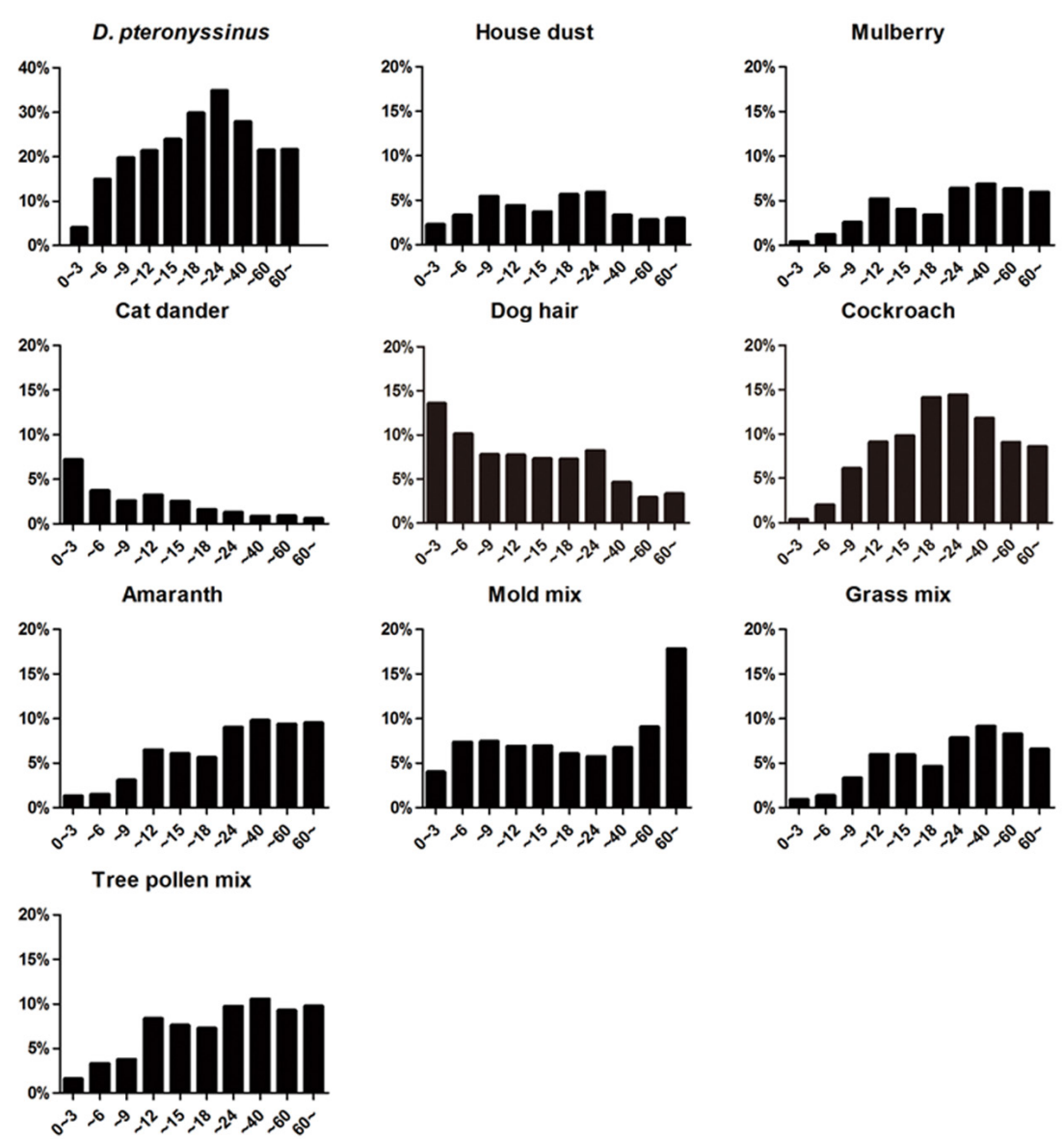

Figure 2. Sensitization to inhaled allergens in individuals of different age groups. The total number of cases in each age group: $0-3$ years $(\mathrm{n}=751), 4-6$ years $(\mathrm{n}=1265), 7-9$ years $(\mathrm{n}=1197), 10-12$ years $(\mathrm{n}=957), 13-15$ years $(\mathrm{n}=591), 16-18$ years $(\mathrm{n}=496)$, $19-24$ years $(\mathrm{n}=1097), 25-40$ years $(\mathrm{n}=3663), 41-59$ years $(\mathrm{n}=3890)$, and 60 years and above $(\mathrm{n}=1627)$.

Egg
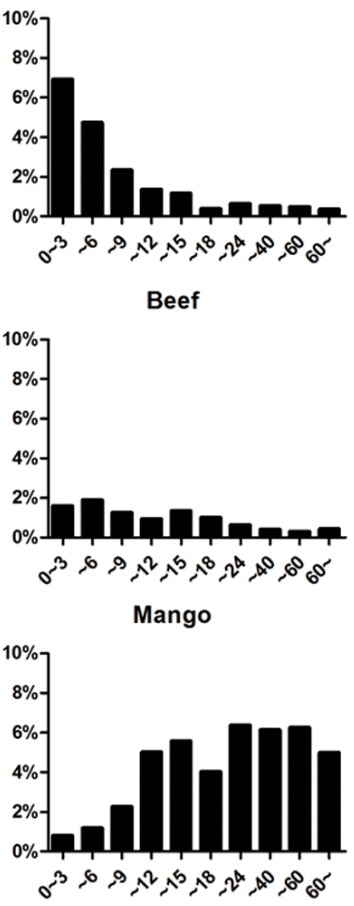

Cow's milk
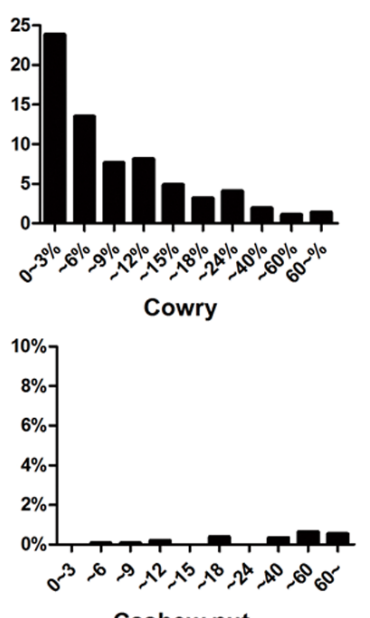

Cashew nut

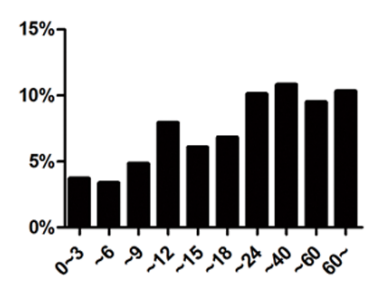

Shrimp
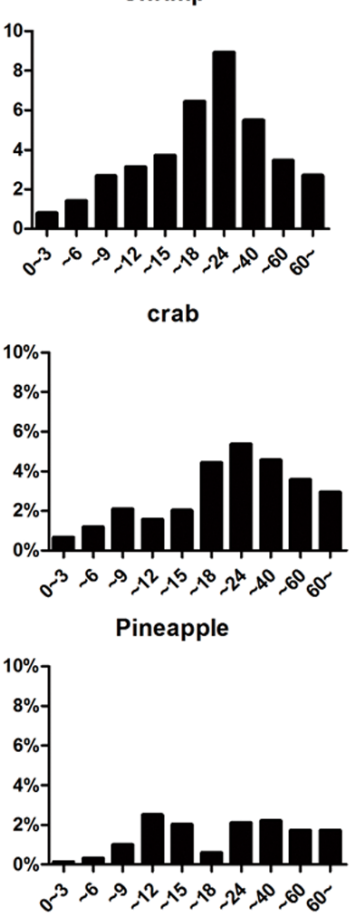

Figure 3. Sensitization to food allergens in individuals of different age groups. The total number of cases in each age group: 0-3 years $(\mathrm{n}=751), 4-6$ years $(\mathrm{n}=1265), 7-9$ years $(\mathrm{n}=1197), 10-12$ years $(\mathrm{n}=957), 13-15$ years $(\mathrm{n}=591), 16-18$ years $(\mathrm{n}=496), 19-24$ years $(n=1097), 25-40$ years $(n=3663), 41-59$ years $(n=3890)$, and 60 years and above $(n=1627)$. 
with an area of $160,000 \mathrm{~m}^{2}$, has a population of more than 107 million, $48.5 \%$ of which were urban population. Zhengzhou is the capital of Henan Province which has a population of more than 9.5 million, $60.8 \%$ of which were urban population. However, the prevalence and distribution of allergen sensitization in this region is unknown until now. To our knowledge, this is the first study to investigate the prevalence of allergen sensitization in recent years in Henan Province, a central region of China.

Our results indicated that $41.97 \%$ (6520) of the subjects were sIgE positive to at least one of the 19 tested allergens. The prevalence of reactivity to inhaled allergens ranged from 1.77$22.79 \%$, while that to food allergens ranged from $0.33-8.50 \%$ in this region. The overall allergen sensitization was reported $33.1 \%$ in a north region of China and $69.1 \%$ in a south region of China. ${ }^{14,15}$ It seems that the overall allergen sensitization is higher in Henan province than that in north China but lower than that in south China. But the tested allergens sources were not so identical among these studies, we should not compare the difference directly.

Among the 19 tested allergens, individuals reacted more often to the inhaled allergen D. pteronyssinus, cockroach and mold mix (Table 1), which is consistent with the report of the previous study. ${ }^{16}$ However, cashew nut and mango were found to be the most frequently tested food allergens in this region which is inconsistent with other studies which reported that egg and cow's milk were the most frequently tested food allergens in China. ${ }^{14,15}$ This results might be due to the difference in study population subsets, this study included more adults than children (66.16\% vs $33.84 \%)$. Food allergic diseases occur more frequently in younger individuals, especially in children under 6 years of age. ${ }^{17}$ Our study also indicated that the most frequently tested food allergens among children below 6 years of age were cow's milk (15.97\%) and eggs (5.00\%), respectively (Table 3). Sensitization to cashew nuts in this study seems unexpectedly high especially in adults. In general, babies and young children are prevented from eating nuts to avoid asphyxia. Less contact may lead less allergic diseases occurrence in children, less contact in children may also lead failure of tolerance induction in older age. This may be one of the reasons, the exact reason remains need further studies. Although seafood is consumed more frequently, certain seafood such as cowry are not common in Henan Province. This might be why the sIgE positive rate for cowry in this region was as low as $0.33 \%$ (Table 1 ).

There were studies reported that allergic diseases appear to be more frequently diagnosed in females than in males. ${ }^{18-20}$ But in this study, the results indicated that in this region male patients were more likely to be sensitive to allergens, and the sIgE positive rates of all individual allergens except cowry and mold mix were higher in males than females (Figure 1). This result was consistent with the findings in two previous studies. ${ }^{21,22}$ However, further studies should be conducted to explain the gender-related difference in allergen sensitization.

At least, three factors are required for the development of allergic disease: allergens, a sensitized host and close contact between them. ${ }^{23}$ Age group analysis in this study revealed that the most frequently tested inhaled allergens in the 0-3, 4-6 groups were cat dander and dog hair, in the 7-9, 10-12, 13-15, 16-18, 19-24, 25-40 groups were D. pteronyssinus and dog hair, in the 41-60 group were D. pteronyssinus and cockroach, and in the over 60 age group was D. pteronyssinus and mold mix (Figure 2). Babies have limited contact with allergens, but encounter more allergens with age. Therefore, the rates of sIgE reactivity to inhaled allergens such as $D$. pteronyssinus and house dust increased from the younger groups to older groups, peaking in the 19-24 or 25-40 age groups. With the acquisition of immune tolerance, they had a descending tendency (Figure 2). The sIgE positive rates for cat dander and dog hair were high in the younger age groups, perhaps because children are more likely to be in close contact with pets as they like to play with them.

Cow's milk and eggs have been reported to be the primary food allergens in children worldwide, including in China. ${ }^{24,25}$ In this study, Cow's milk and eggs were also found the most frequently tested food allergens in the early age groups. As cow's milk is the earliest food given to babies, followed by eggs, and these are both commonly consumed foods in daily life. Therefore, sensitization to egg and cow's milk primarily occurs in infants and young children.

Several previous studies have investigated the incidence of allergen sensitization for allergic disease in Chinese populations. ${ }^{14,20,26}$ However, this is the first large-scale study to report the prevalence of allergen sensitization among patients with suspected allergic diseases in Henan Province, a central region of China. The finding in this study may also be applicable in other provinces in the central region of China which have similar climates, lifestyles to Henan, but further studies are needed to provide evidence. However, the present study has several limitations. First, sampling error may exist in the study as all the patients enrolled in this study were not clinically diagnosed cases but had symptoms typical of allergies, and were suspected to have allergies, the overall positivity reactivity $41.97 \%$ we got may be lower than the real positivity reactivity in patients with allergic diseases in this region. Second, many allergens present in the environment, reactivity to only 19 allergens was tested. ${ }^{27}$ Meanwhile the difference in tested allergen sources would also limit the capability to compare with other studies. Furthermore, there may be bias due to the retrospective nature of this study. Further prospective studies should be conducted on a larger scale to determine the incidence and prevalence of allergen sensitization in China.

In conclusion, the prevalence and distribution of allergens differed in gender and age groups in Henan Province, a central region of China. The characteristics of allergen sensitization revealed in this study would facilitate the prevention, diagnosis, and management of allergic diseases in this region.

\section{Conflict of interest}

The authors declare no conflict of interest.

\section{Acknowledgments}

This work was supported by the National Natural Science Foundation of China (No.81501715) and the Key Project on Science and Technology Research provided by Henan Province, China(No.152102410067). 


\section{References}

1. Loftus PA, Wise SK. Epidemiology of asthma. Curr Opin Otolaryngol Head Neck Surg. 2016;24:245-9.

2. Turner PJ, Gowland MH, Sharma V, Ierodiakonou D, Harper N, Garcez $\mathrm{T}$, et al. Increase in anaphylaxis-related hospitalizations but no increase in fatalities: an analysis of United Kingdom national anaphylaxis data, 1992-2012. J Allergy Clin Immunol. 2015;135:956-63.e1.

3. Mullins RJ, Dear KB, Tang ML. Time trends in Australian hospital anaphylaxis admissions in 1998-1999 to 2011-2012. J Allergy Clin Immunol. 2015;136: 367-75.

4. Meltzer EO. Allergic Rhinitis: Burden of Illness, Quality of Life, Comorbidities, and Control. Immunol Allergy Clin North Am. 2016;36: 235-48.

5. Antolín-Amérigo D, Manso L, Caminati M, de la Hoz Caballer B, Cerecedo I, Muriel A, et al. Quality of life in patients with food allergy. Clin Mol Allergy. 2016;14:4.

6. Liu X, Zhang S, Tsai HJ, Hong X, Wang B, Fang Y, et al. Genetic and environmental contributions to allergen sensitization in a Chinese twin study. Clin Exp Allergy. 2009;39:991-8.

7. Li J, Maggadottir SM, Hakonarson H. Are genetic tests informative in predicting food allergy. Curr Opin Allergy Clin Immunol. 2016;16:257-64.

8. de Vos G. Skin testing versus serum-specific IgE testing: which is better for diagnosing aeroallergen sensitization and predicting clinical allergy. Curr Allergy Asthma Rep. 2014;14:430.

9. Smits WL, Letz KL, Evans TS, Giese JK. Evaluating the response of patients undergoing both allergy skin testing and in vitro allergy testing with the ImmunoCAP Technology System. J Am Acad Nurse Pract. 2003;15: 415-23.

10. Linneberg A. The increase in allergy and extended challenges. Allergy. 2011;66 Suppl 95: S1-3.

11. El-Ghitany EM, Abd EMM. Environmental intervention for house dust mite control in childhood bronchial asthma. Environ Health Prev Med. 2012;17: 377-84.

12. Du Toit G, Foong RM, Lack G. Prevention of food allergy - Early dietary interventions. Allergol Int. 2016; 65:370-377.

13. Halken S. Prevention of allergic disease in childhood: clinical and epidemiological aspects of primary and secondary allergy prevention. Pediatr Allergy Immunol. 2004;15 Suppl 16:S4-5, 9-32.

14. Chang ML, Shao B, Liu YH, Li LL, Pei LC, Wang BY. Analysis of allergens in 5473 patients with allergic diseases in Harbin, China. Biomed Environ Sci. 2013;26:886-93.
15. Sun BQ, Zheng PY, Zhang XW, Huang HM, Chen DH, Zeng GQ. Prevalence of allergen sensitization among patients with allergic diseases in Guangzhou, Southern China: a four-year observational study. Multidiscip Respir Med. 2014;9:2

16. Huss K, Adkinson NF, Eggleston PA, Dawson C, Van Natta ML, Hamilton RG. House dust mite and cockroach exposure are strong risk factors for positive allergy skin test responses in the Childhood Asthma Management Program. J Allergy Clin Immunol. 2001;107:48-54.

17. Sampson HA. Food allergy: Past, present and future. Allergol Int. 2016; 65(4):363-369. Epub 2016 Sep 6.

18. Kynyk JA, Mastronarde JG, McCallister JW. Asthma, the sex difference. Curr Opin Pulm Med. 2011;17:6-11.

19. Cassano N, Colombo D, Bellia G, Zagni E, Vena GA. Gender-related differences in chronic urticaria. G Ital Dermatol Venereol. 2016;151:544-52.

20. Mao D, Tang R, Wu R, Hu H, Sun LJ, Zhu H, et al. Prevalence trends in the characteristics of patients with allergic asthma in Beijing, 1994 to 2014. Medicine (Baltimore). 2017;96:e7077.

21. Stemeseder T, Klinglmayr E, Moser S, Lang R, Himly M, Oostingh GJ, et al. Influence of Intrinsic and Lifestyle Factors on the Development of IgE Sensitization. Int Arch Allergy Immunol. 2017;173:99-104.

22. Scala E, Alessandri C, Palazzo P, Pomponi D, Liso M, Bernardi ML, et al. IgE recognition patterns of profilin, PR-10, and tropomyosin panallergens tested in 3,113 allergic patients by allergen microarray-based technology. PLoS One. 2011;6:e24912.

23. Kozyrskyj AL, Bahreinian S, Azad MB. Early life exposures: impact on asthma and allergic disease. Curr Opin Allergy Clin Immunol. 2011;11: 400-6.

24. Ramesh S. Food allergy overview in children. Clin Rev Allergy Immunol. 2008;34:217-30.

25. Liu XJ, Zhu TT, Zeng R, Chang L, Li FY, Li WS, Jiang YM. An epidemiological study of food intolerance in 2434 children. Zhongguo Dang Dai Er Ke Za Zhi. 2013;15:550-4. Chinese.

26. Leung R, Ho P, Lam CW, Lai CK. Sensitization to inhaled allergens as a risk factor for asthma and allergic diseases in Chinese population. J Allergy Clin Immunol. 1997;99:594-9.

27. Boyce JA, Assaad A, Burks AW, Jones SM, Sampson HA, Wood RA, et al. Guidelines for the diagnosis and management of food allergy in the United States: summary of the NIAID-sponsored expert panel report. Nutr Res. 2011;31:61-75. 